\title{
Renal artery aneurysm rupture during post-partum period: A case report
}

\author{
Hassan Ravari ${ }^{1}$, Atiyeh Vatanchi ${ }^{2}$, Leila Pourali ${ }^{2}$, Malihe Afarideh ${ }^{3}$, Salmeh Dadgar ${ }^{2}$
}

\begin{abstract}
${ }^{1}$ M.D., Associate Professor of Vascular Surgery, Department of Vascular Surgery, Imam Reza Hospital, Faculty of Medicine, Mashhad University of Medical Sciences (MUMS), Mashhad, Iran

${ }^{2}$ M.D., Assistant Professor of obstetrics and Gynecology, Department of Obstetrics and Gynecology, Faculty of Medicine, Mashhad University of Medical Sciences (MUMS), Mashhad, Iran

${ }^{3}$ M.D., Resident of Obstetrics and Gynecology, Department of Obstetrics and Gynecology, Faculty of Medicine, Mashhad University of Medical Sciences (MUMS), Mashhad, Iran
\end{abstract}

\section{Type of article: Case report}

\begin{abstract}
Renal artery aneurysm has an incidence of lower than one in 1000 of the general population. The risk of aneurysm rupture increases during pregnancy due to hormonal and hemodynamic changes. Therefore, these patients often refer to physicians with abdominal pain that is followed by shock. We report the case of a 41 -yearold multiparous woman who was referred with symptoms of epigastric pain and hemorrhagic shock, a week after her vaginal delivery. She was diagnosed with renal artery aneurysm in ultrasonography. The patient underwent laparotomy and nephrectomy so that she was discharged from hospital with a good general health condition. Imaging measures in a timely manner, carried out by qualified individuals considering a patient's history and clinical situation is very effective in diagnosis and treatment of post-partum abdominal pain.

Keywords: Ruptured Aneurysms, Puerperium, Pregnancy
\end{abstract}

\section{Introduction}

During pregnancy, following hemodynamic and endocrine changes and increased intra-abdominal pressure, arterial changes might lead to forming a new aneurysm or weakening the pre-existing aneurysm's wall. Aneurysms are more frequent in aorta, cerebral, splenic, renal, coronary, and ovarian arteries. The incidence of renal artery aneurysm occurrence is lower than $1 / 1000$ (1). It is usually discovered in the general population either accidentally or after searching for hypertension (2). Ruptured arterial aneurysms are more likely to happen during pregnancy compared to the postpartum period. The prognosis of ruptured renal aneurysms is poor and it is associated with kidney loss (3). Hence, early detection and timely treatment can increase mother and newborn's survival. Our case is a renal aneurysm rupture in post-partum period that is very rare.

\section{Case presentation}

\subsection{Clinical presentation and medical history}

The patient is a 41-year-old G5L3Ab1 woman. She was 38 weeks pregnant when she referred to one of the women's teaching hospitals, affiliated to Mashhad University of Medical Sciences in April, 2016. She did not have any history of medical diseases and the only medications she consumed were pregnancy supplements. She had a 6-7 cm dilation. After about two hours, vaginal delivery was carried out without therapeutic intervention and the healthy baby was born with Apgar of 9-10 and weight of $3100 \mathrm{~g}$. Thus, the patient, who was in a good general health condition, was discharged from the hospital the following day. After three days, the patient reported suffering from progressive weakness and fatigue. On the seventh day after birth, the patient suffered from epigastric pain and vomiting, so that she felt dizzy and fainted at home. The patient was referred to the same hospital by ambulance. Although she was conscious when admitted, her conjunctiva was pale, her skin was cold and she sweated

\section{Corresponding author:}

Assistant Professor Dr. Atiyeh Vatanchi, Department of Obstetrics and Gynecology, Faculty of Medicine, Mashhad University of Medical Sciences (MUMS), Mashhad, Iran. Tel: +98.5138012477, Email: vatanchia@mums.ac.ir Received: July 13, 2016, Accepted: November 16, 2016, Published: August 2017 iThenticate screening: August 30, 2016, English editing: March 12, 2017, Quality control: June 17, 2017

(C) 2017 The Authors. This is an open access article under the terms of the Creative Commons Attribution-NonCommercialNoDerivs License, which permits use and distribution in any medium, provided the original work is properly cited, the use is non-commercial and no modifications or adaptations are made. 
excessively. Vital signs were found as SBP 55 / PR: 100 / RR: 25. The abdomen was soft with tenderness and rebound. The patient had right Costovertebral Angle Tenderness (CVAT). The abdominal mass was not touched and intestines were projected to the abdominal wall. Uterine size and vaginal bleeding were at the normal level of postpartum period. No urological and gastrointestinal symptoms were observed. After administration of about 3.5 liters of Ringer's lactate and normal saline, the patient's blood pressure reached to 95.60 and $\mathrm{u} / \mathrm{o}$ : $30 \mathrm{cc} / \mathrm{h}$ was established.

\subsection{Laboratory tests and imaging}

In the early experiments, HCT: 24 HB: 8 and WBC: 23000 were recorded. Additionally, U/A microscopic hematuria was at blood ${ }^{+}$. Since there was no sign of abdominal pain and normal examination of the abdomen was carried out, the patient underwent bedside sonography. Clotting and dispersed fluid were reported in the abdominal cavity. A cystic focus $(50 \mathrm{~mm})$ was reported in the right kidney with a turbulent flow suggesting the occurrence of renal artery aneurysm. During her transfer from the radiology ward, the patient underwent a sudden pressure drop to $60 / 40$ mmHg, excessive sweating (hyperhidrosis), and PR 140/min.

\subsection{Treatment}

The patient was immediately transferred to the operating room where the midline abdominal incision was made. A massive extension of hematoma (zone 2) of the abdomen in the right side was explored (Figure 1). After exploration and trying for proximal control, aneurysmectomy and nephrectomy was performed for the patient (Figure 2). Aortic repair was done by prolene 3-0. During the surgery, the patient received 11 units of packed cell, 10 units of Platelet, and 10 units of Fresh Frozen Plasma (FFP). After extubation at the operating room, the patient was transferred to ICU. Enoxaparin was initiated and the patient was PO after 36 hours. The patient was discharged from the hospital after 6 days. She returned to her daily life with a good general health condition and normal functioning of the remaining kidney.

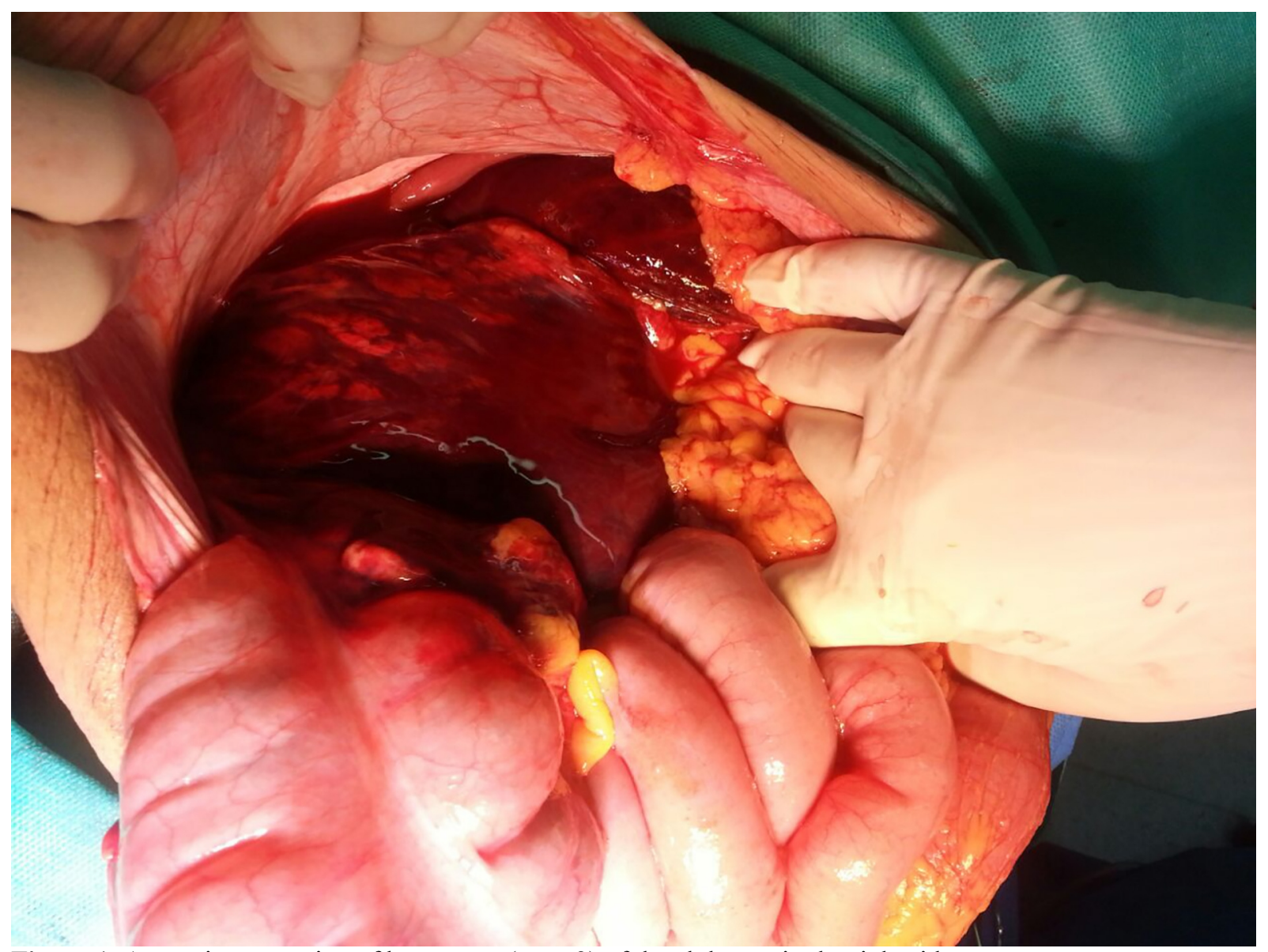

Figure 1. A massive extension of hematoma (zone 2) of the abdomen in the right side 


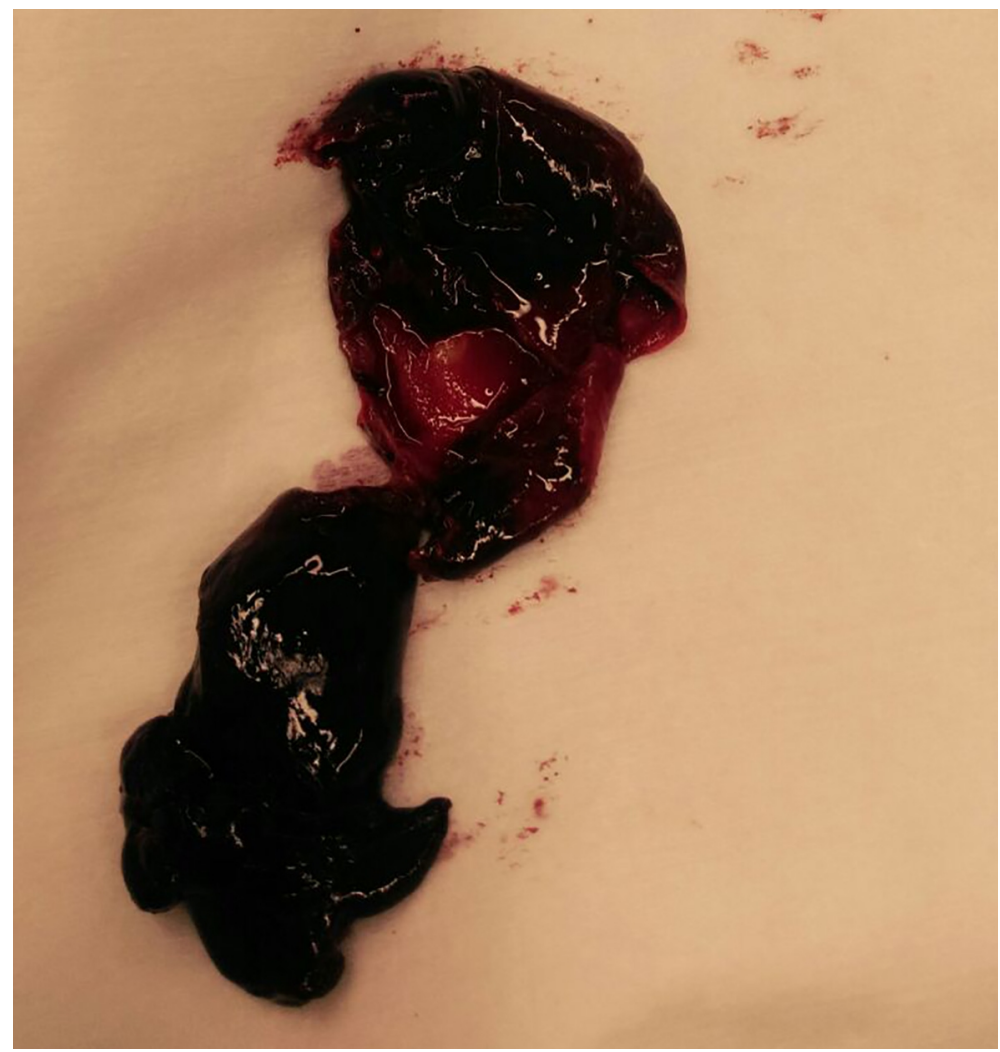

Figure 2. Right kidney and its aneurysm

\section{Discussion}

Aneurysms have some risk factors such as atherosclerosis, fibromuscular dysplasia, trauma, or iatrogenic damages. Spontaneous rupture of the aneurysm is uncommon. The risk factors for the rupture of aneurysm include size $>2 \mathrm{~cm}$, hypertension, and pregnancy (4). Renal artery aneurysm incidence is very low and is usually asymptomatic. It is commonly discovered randomly through conducting surveys for hypertension. Sometimes, only one bruit can be discovered on the upper quadrant of the abdomen at the hearing. Although microscopic hematuria is observed in 10$40 \%$ of cases, the kidney functions normally. Abdominal or back pain are the clinical symptoms of ruptured renal aneurysm. This case had no past medical history of hypertension, and recent pregnancy was the only risk factor of aneurysm. She had no sign or symptom of renal aneurysm before its rupture. There seems to be no relationship between maternal age and parity with aneurysm rupture. Ruptured aneurysms prognosis remains poor and associates with the loss of patients' kidney (5). In most reported cases of ruptured renal aneurysm, nephrectomy was inevitable but mortality was dependent on early diagnosis and a skilled surgeon. At first, we thought it was a puerperal complication, particularly uterine hematoma, but sonography diagnosis of renal aneurysm led to the vascular surgeon asking for help. This pre-operation diagnosis surely improved her prognosis. In cases of Aneurysm rupture and hematoma formation during pregnancy, the uterus may be shifted to the opposite side. In this case, retroperitoneal massive hematoma had led to a shift and projection of the intestines to the abdominal wall. Based on clinical manifestation, Aneurysm rupture begins with an initial pain and it follows the symptoms of hemorrhagic shock after the progress of rupture to retroperitoneal space. Rupture of the renal artery aneurysm (RAA) during pregnancy, usually occurs in the third trimester and at the left side (6). In a study carried out by Maughan (1), Aneurysm rupture was manifested in a 22 -week pregnant woman with severe abdominal pain, shock, and fetal death. In another study, aneurysm rupture was observed one day after caesarean section, with gross hematuria manifestation following CT angiography (4). Different treatments are available for RAA in pregnant and postpartum patients. According to clinical manifestations and patients' vital signs, these methods include; monitoring, renal embolization, and surgical procedures. In intraparenchymal and hilar aneurysm of kidney, nephrectomy is the most secure way for a patient, which was performed on our patient too. Since this patient had unstable vital signs, there was not enough opportunity to perform other imaging techniques and embolization. In similar cases, patients who refer to us with symptoms of shock in postpartum, a large list of differential diagnoses arises. Among all, the issues related to postpartum period such as pelvic hematoma have the most priority. Other differential diagnoses are 
ovarian and urologic complications. If patients' hemodynamic condition is stable, ultrasound imaging can be carried out. However, ultrasonography might not be able to properly identify the retroperitoneal pathology. Presence of a qualified radiologist, consultation with the gynecologist and anesthetist, and consideration of the patient's clinical manifestations are helpful in diagnosing in a timely manner. Admission to a third-level hospital, as well as a quick access to radiology, vascular surgeon, and blood bank is very crucial and helpful in saving a patients' life. Patients are advised to examine angiography for all arteries. Treatment is not often required if there is an aneurysm in the contralateral kidney, discovered by accident. Frequent follow-ups suggest that these patients are not at high risk. However, treatment threshold in childbearing age women should be lower due to high probability of aneurysm rupture during pregnancy.

\section{Conclusions}

During pregnancy and postpartum period, in cases of any instability in vital signs, vascular diagnoses such as rupture of aneurysms must be taken into account in addition to the midwifery diagnoses. In the case of approving diagnosis of visceral aneurysms complications based on patient's condition and after consultation with vascular surgeons, the patient can be treated. Lack of attention to the differential diagnoses and delay in treatment can cause mortality.

\section{Acknowledgments:}

We hereby wish to thank Dr. Izadi for his great help in the sonography of our patient that led to early diagnosis of aneurysm.

\section{Conflict of Interest:}

There is no conflict of interest to be declared.

\section{Authors' contributions:}

All authors contributed to this project and article equally. All authors read and approved the final manuscript.

\section{References:}

1) Maughan E, Webster C, Konig T, Renfrew I. Endovascular management of renal artery aneurysm rupture in pregnancy - A case report. Int J Surg Case Rep. 2015; 12: 41-3. doi: 10.1016/j.ijscr.2015.05.011. PMID: 25996776, PMCID: PMC4486101.

2) Lacroix H, Bernaerts P, Nevelsteen A, Hanssens M. Ruptured renal artery aneurysm during pregnancy: successful ex situ repair and autotransplantation. J Vasc Surg. 2001; 33(1): 188-90. doi: 10.1067/mva.2001.109767. PMID: 11137943.

3) Enakpene CA, Stern T, Barzallo Salazar MJ, Mukherjee P. Spontaneous Rupture of an Ovarian Artery Aneurysm: A Rare Postpartum Complication. Case Rep Obstet Gynecol. 2016; 2016: 1029561. doi: 10.1155/2016/1029561. PMID: 27034862, PMCID: PMC4789400.

4) Hwang PF, Rice DC, Patel SV, Mukherjee D. Successful management of renal artery aneurysm rupture after cesarean section. J Vasc Surg. 2011; 54(2): 519-21. doi: 10.1016/j.jvs.2010.12.041. PMID: 21316184.

5) Parpaglioni R, Metta E, Zagari A, Celleno D. Spontaneous splenic vein aneurysm rupture in the puerperium. International journal of obstetric anesthesia. 2009; 18(1): 48-51. doi: 10.1016/j.ijoa.2008.01.019. PMID: 18684614.

6) Rijbroek A, van Dijk HA, Roex AJ. Rupture of renal artery aneurysm during pregnancy. Eur J Vasc Surg. 1994; 8(3): 375-6. doi: 10.1016/S0950-821X(05)80162-8. PMID: 8013695. 